\title{
A smart power system restoration based on the merger of two different strategies
}

DOI:

10.1109/ISGTEurope.2012.6465623

Link to publication record in Manchester Research Explorer

\section{Citation for published version (APA):}

Quiros Tortos, J., \& Terzija, V. (2012). A smart power system restoration based on the merger of two different strategies. In IEEE PES Innovative Smart Grid Technologies Conference Europe/IEEE PES Innovative. Smart Grid Technol. Conf. Europe IEEE. https://doi.org/10.1109/ISGTEurope.2012.6465623

\section{Published in:}

IEEE PES Innovative Smart Grid Technologies Conference Europe|IEEE PES Innovative. Smart Grid Technol. Conf. Europe

\section{Citing this paper}

Please note that where the full-text provided on Manchester Research Explorer is the Author Accepted Manuscript or Proof version this may differ from the final Published version. If citing, it is advised that you check and use the publisher's definitive version.

\section{General rights}

Copyright and moral rights for the publications made accessible in the Research Explorer are retained by the authors and/or other copyright owners and it is a condition of accessing publications that users recognise and abide by the legal requirements associated with these rights.

\section{Takedown policy}

If you believe that this document breaches copyright please refer to the University of Manchester's Takedown Procedures [http://man.ac.uk/04Y6Bo] or contact uml.scholarlycommunications@manchester.ac.uk providing relevant details, so we can investigate your claim.

\section{OPEN ACCESS}




\title{
A Smart Power System Restoration Based on the Merger of Two Different Strategies
}

\author{
Jairo Quirós Tortós, Student Member, IEEE, Vladimir Terzija, Senior Member, IEEE
}

\begin{abstract}
Power System Restoration (PSR) is carried out after a partial or complete blackout of a network. Its objective is to restore the power system as quickly as possible while satisfying all the constraints previously defined by grid codes. Two main strategies are currently used worldwide. The "buildup" strategy sectionalises the power system affected by the blackout. This strategy creates small subsystems and then applies parallel power system restoration. On the other hand, the "build-down" strategy preserves the complete network structure and energises the skeleton of the system. The decision of which strategy is undertaken depends mainly on the network topology. As both strategies have their advantages, this paper proposes to merge these in order to be benefited by both and, therefore, obtain an improved PSR. The proposed methodology can improve the restoration process of large scale networks affected by a complete blackout. The proposed methodology is implemented and validated on the IEEE 118bus test system. Results from these studies demonstrate the effectiveness of the method to restore the network quickly.
\end{abstract}

Index Terms--Build-down strategy, build-up strategy, parallel power system restoration, power system restoration, transmission system restoration.

\section{INTRODUCTION}

$\mathrm{P}$ OWER System Restoration (PSR) is the process carried out after a partial or complete blackout. Restoring the entire power system as quickly as possible, taking into consideration static and dynamic constraints previously defined by grid codes, is the main objective of power system operators during the restoration process. By speeding up the restoration process, operators directly reduce the cost of the power system blackout; thus, increasing the importance to speed up the restoration process.

Since PSR is a long and complex task, it is commonly divided into three different steps. These steps and tasks carried out at each step are shown in Fig. 1. As noted, the first task during this process is to assess the status of the perturbed network. This is followed by the reconnection of various generation units, the subsequent identification of restoration paths and the load pick up, in which loads are reconnected in small increments to avoid excessive frequency deviations [1], [2].

The average duration of these fundamental steps is shown in Fig. 2 [3]. As noticed, the restoration process is long and can last hours, and in multiple cases, days [4], [5]. The restoration delay noticed in Fig. 2 is normally caused by the

This work was supported in part by the Engineering and Physical Science Research Council (EPSRC) in the UK and The University of Costa Rica.

J. Quirós Tortós and V. Terzija are with the School of Electrical \& Electronic Engineering, The University of Manchester, Manchester, UK (email: jairoquirostortos@ieee.org, terzija@ieee.org). unknown system status, the identification of the blackout cause and other electrical and mechanical constraints.

PSR can be conceptually viewed as a multi-objective, multi-stage, combinatorial, nonlinear constrained optimization problem which can be modelled as follows.

$$
\begin{array}{cc}
\min _{\mathbf{x}, \mathbf{u}_{\mathbf{c}}} & F\left(\mathbf{x}, \mathbf{u}_{\mathbf{c}}\right) \\
\text { s.t } \quad \dot{\mathbf{x}}=f\left(\mathbf{x}, \mathbf{u}_{\mathbf{c}}, \mathbf{u}_{\mathbf{d}}\right) \\
g\left(\mathbf{x}, \mathbf{u}_{\mathbf{c}}, \mathbf{u}_{\mathbf{d}}\right)=0 \\
\\
h\left(\mathbf{x}, \mathbf{u}_{\mathbf{c}}, \mathbf{u}_{\mathbf{d}}\right) \leq 0
\end{array}
$$

In (1), $F$ represents the objective criterion, $f, g$ and $h$ are nonlinear constraint functions, $\mathbf{x}$ is the vector of dependent systems variables (e.g. voltage magnitudes at load buses, generators power output and frequency), $\mathbf{u}_{\mathbf{c}}$ is the vector of control inputs (e.g. reconnecting system branches and flow limits), and $\mathbf{u}_{\mathbf{d}}$ is the vector of uncontrolled inputs (e.g. load demand and interconnection flows).

Two main strategies to restore the power system are stated in [2] and [6]. The first strategy, the "build-up" strategy, sectionalises the entire power system into smaller subsystems, also called islands, and then restores the created

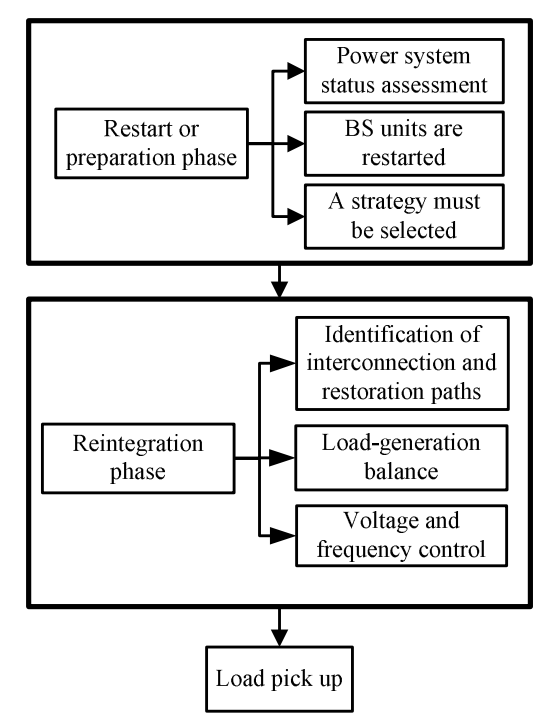

Fig. 1. Power system restoration overview

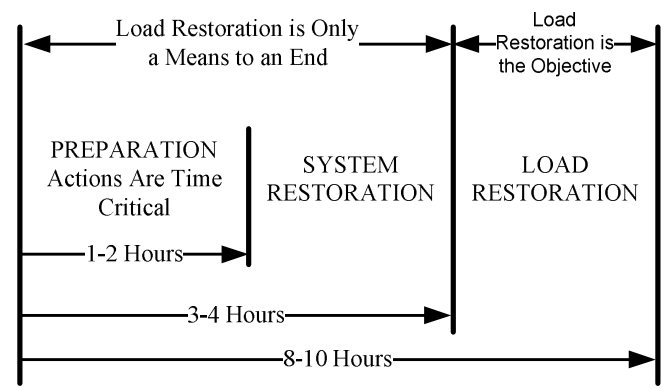

Fig. 2. Typical restoration stages and required time 
islands by applying Parallel Power System Restoration (PPSR). The second approach, the "build-down" strategy, reenergises the bulk network before re-synchronising loads and generators. Since the restoration duration is significantly reduced when using the former strategy, this is considered as the predominantly used worldwide restoration scheme.

As it was previously mentioned, the "build-up" strategy, or simply PPSR, separates the entire power system into electrically isolated islands. When the islands are created, each subsystem is restored in parallel. When all islands are completely stable, these are resynchronised and the entire network is finally restored. Two main advantages of this approach are the restoration time duration, which is considerably reduced, and in case of a large disturbance in an island, a recurrent blackout would only be obtained in the affected area. However, as the network is separated into islands, the stability margins of the islands are an important issue to consider during PPSR. Also, different blackstart (BS) units are required to be available.

On the other hand, during the "build-down" strategy, the main part of the network, also called the skeleton or backbone, must be energised first. Loads and power stations should be energised in parallel. The main advantage of this strategy is that the stability margins are significant. Therefore, the reliability and probability of a successful restoration are very good. The main drawback of the "builddown" strategy is the time needed for restoring the entire network, the high reactive power produced by unloaded high voltage transmission lines, and most importantly, in case of a large disturbance, the entire system would again be affected, leading to a recurrent large scale blackout [6].

Different methodologies, to completely separate the network and apply PPSR or to determine the skeleton of the system and implement the "build-down" strategy are proposed in the literature. For instance, to separate the network, an Ordered Binary Decision Diagram (OBDD) based method considering different constraints i.e. BS availability, load-generation balance, and voltage stability, is proposed in [7]. On the other hand, to reconfigure the backbone, a method based on topological characteristics of scale-free networks and a discrete Particle Swarm Optimization algorithm is proposed in [8]. Also, a Power Transfer Distribution Factor based path selection approach for large scale power systems focused on the load restoration stage is presented in [9].

Although different methods to either sectionalise or to restore the entire network are proposed in the literature, the merger of these two strategies (sectionalisation and then reconfiguration) is proposed for the first time in this paper. By merging these strategies, a better restoration process which combines the advantages of both can be obtained. When selecting and implementing the proposed method, it is important to monitor static and dynamic constraints, such as load-generation balance, transmission line loading, voltage limits and BS capacity, during the restoration process [2]. Therefore, the availability of Phasor Measurement Units (PMUs) is assumed in this paper.

Based on the fact that an improved PSR can be obtained by merging these two strategies, this paper proposes a two step method, sectionalisation and reconstruction of the network, to restore a large scale power system affected by a complete blackout. By representing the network as an Undirected Finite Graph (UFG), the first step of the method separates the large scale network into the fewest islands required to maintain the stability margins as high as possible. These areas are obtained by sectionalising the UFG.

The second step, which is carried out simultaneously for each determined island, is divided into three sub-stages: identification, simulation and implementation. Using the created sub-graphs, an efficient algorithm to determine the $k$-shortest simple paths from the BS units to the nonblackstart (NBS) units and critical loads (CLs) in the same island is performed in the first sub-stage. Then, these paths are ranked based on the number of connections required to energise the element, the electrical distance to the NBS units or CLs, and the steady-state voltage magnitude obtained at the bus where this element is connected. Since the restoration time duration must be minimised to reduce the blackout cost, the number of connections required (which is proportional to the time duration) is represented as a high weight factor.

In the final sub-stage i.e. the implementation, the optimal option is selected and implemented in each island. When implementing the path with the minimum weight factors, the use of PMUs installed across the power systems is essential to monitor and to help in the control of high voltages, unexpected large charging currents and other phenomena that might arise during PSR. Therefore, the proposed methodology could be potentially supported by Wide Area Monitoring Protection and Control applications. Since the number of elements to connect the remaining elements might change, the shortest simple paths list is updated after implementing each optimal path.

The main contributions of this paper are:

- A two step method to restore a power system affected by a complete blackout is presented. The merger of two different PSR strategies in order to improve the restoration process is introduced.

- An efficient algorithm to determine the $k$-shortest simple paths from BS units (source nodes) to NBS units and CLs (destination nodes) is introduced.

- An OPF to reduce active power losses, in order to be benefited of sufficient power capacity in branches during PSR is presented. Also, an optimal steady-state voltage is obtained at all of the buses.

The main body of the paper is organised as follows. Section II presents different PSR strategies. Section III explains the proposed methodology in detail. Section IV presents the results simulations. Finally, a conclusion drawn from the analysis is given in Section V.

\section{Power System Restoration StRategies}

Despite the blackout causes, it is important to note that the strategy to be carried out during PSR depends on a number of factors such as the inherent system topology, the size of the network, and the location and capacity of BS units and emergency backup power supplies [2].

The two main methodologies explained in Section I were firstly mentioned in [2]. This paper also details the restoration plans for thermal systems and hydro-thermal systems. It is mentioned in [2] that hydro-thermal systems 


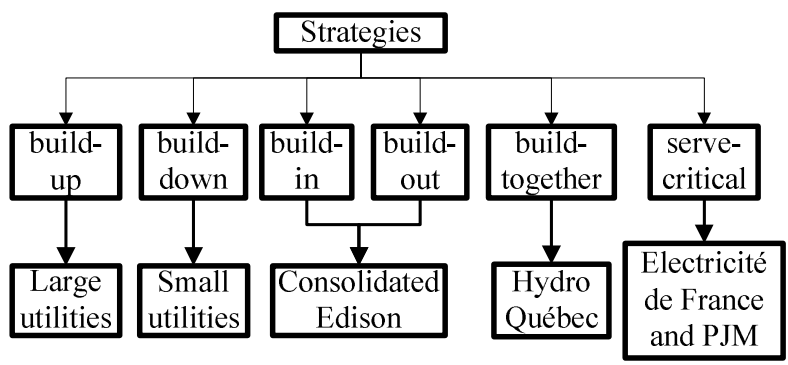

Fig. 3. Power system restoration strategies

take advantage of large hydro stations to absorb the charging currents of the complete transmission systems. Other less common restoration strategies ("build-in", "build-out", "build together" and "serve-critical"), based on the strategies performed worldwide, are introduced in [10]. Utilities performing these restoration strategies can be visualised in Fig. 3. However, most of them have based their restoration process on the main two with small adaptations depending on the factors mentioned previously.

\section{Merging Two DifFerent Strategies}

The steps previously mentioned to restore a power system are commonly taken as a reference. Extra steps are normally added in order to obtain the optimal PSR according to each network and utilities needs.

Since multiple power systems properties can be conveniently described by means of a diagram consisting of a set of vertices together with a set of edges joining certain pairs of these vertices, this paper models a power system with $n_{b}$ buses, $n_{l}$ branches and $n_{g}$ generators as an undirected finite graph $\mathbf{G}=(\mathbf{V}, \mathbf{E}, \mathbf{U}, \mathbf{W})$. Here, the elements $v_{i} \in \mathbf{V}$, $i=1,2, \ldots, n_{b}$, and $e_{i} \in \mathbf{E} \subset \mathbf{V} \times \mathbf{V}, i=1,2, \ldots, n_{l}$, denote the nodes and edges of the graph, respectively. The sets $\mathbf{V}$ and $\mathbf{E}$ represent the buses and branches of the actual power system, respectively. The elements $e_{i} \in \mathbf{E}$ are unordered pairs $i, j$ of buses denoted as $(i, j) \in \mathbf{E}$. A subset $\mathbf{V}_{\mathbf{G}} \subset \mathbf{V}$ is defined to represent the generators available during PSR. This subset also contains the BS units. The set $\mathbf{U}$ with elements $u_{i} \in \mathbf{U}$, $i=1,2, \ldots, n_{b}$, contains the weight factors associated to each node. These weight factors are computed as shown in (2). Here, $P_{G}$ and $P_{L}$ are the generated and consumed active power at the bus, respectively. The nonnegative symmetric length from two connected nodes $i$ and $j$ is given to each edge and it is contained in the set $\mathbf{W}$. These weight factors $w_{i} \in \mathbf{W}, i=1,2, \ldots, n_{l}$, are defined as shown in (3).

$$
\begin{gathered}
u_{i}=P_{G}^{i}-P_{L}^{i} \\
w_{i}= \begin{cases}1 & \text { if } i \text { is connected to } j \\
\infty & \text { otherwise }\end{cases}
\end{gathered}
$$

The proposed method is carried out in two steps. The separation of the power system after the blackout into the fewest islands required to maintain the stability margins as high as possible is carried out first. When the branches to be left disconnected to create the islands are known, three substages (identification, simulations and implementation) are then carried out in the second step. The two step method is detailed in the next two subsections.

\section{A. First step: sectionalising the network}

When a large scale power system is affected by a complete blackout, the power system status assessment is carried out first. In the first step, the proposed methodology identifies the set of branches to be left disconnected in order to create $n$ different and electrically isolated islands. By separating the system into the fewest possible number of islands i.e. by defining $n$ as small as possible, the restoration process is speeded up and high stability margins are maintained. In order to determine the sectionalising strategy to separate the network, this paper implements the OBDD based method presented in [7].

The OBDD based method presented in [7] considers three main constraints. These must be taken into consideration when sectionalising the network into subsystems after a blackout. The first constraint is the BS constraint $(B S C)$, which ensures the availability of at least one BS unit within each island. The second is the power balance constraint $(P B C)$, which ensures the ability to match generation and load to a prescribed tolerance within each island. Finally, the voltage stability constraint (VSC) excludes from the sectionalising strategy the critical lines that maintain system voltage stability [7].

Given an undirected and connected graph $\mathbf{G}=(\mathbf{V}, \mathbf{E}, \mathbf{U}, \mathbf{W})$, the OBDD based method separates $\mathbf{G}$ into two disjoint sub-graphs $\mathbf{G}_{\mathbf{1}}\left(\mathbf{V}_{\mathbf{1}}, \mathbf{E}_{\mathbf{1}}, \mathbf{U}_{\mathbf{1}}, \mathbf{W}_{\mathbf{1}}\right)$ and $\mathbf{G}_{\mathbf{2}}\left(\mathbf{V}_{\mathbf{2}}, \mathbf{E}_{\mathbf{2}}, \mathbf{U}_{\mathbf{2}}, \mathbf{W}_{\mathbf{2}}\right)$. Since $\mathbf{G}_{\mathbf{1}}$ and $\mathbf{G}_{\mathbf{2}}$ are disjoint, $\mathbf{G}_{\mathbf{1}} \cup \mathbf{G}_{\mathbf{2}}=\mathbf{G}$ and $\mathbf{G}_{\mathbf{1}} \cap \mathbf{G}_{\mathbf{2}}=\varnothing$. The $B S C$ is ensured by defining two sub-sets $\mathbf{V}_{\mathbf{G A}}$ and $\mathbf{V}_{\mathbf{G S}}$ such that $\mathbf{V}_{\mathbf{G A}} \subset \mathbf{V}_{\mathbf{1}}$ and $\mathbf{V}_{\mathbf{G S}} \subset \mathbf{V}_{\mathbf{1}}$. The $P B C$ is then satisfied by computing (4).

$$
\left|\sum_{\forall v_{i} \in \mathbf{V}_{1}} u_{i}\right| \leq \varepsilon, \quad\left|\sum_{\forall v_{j} \in \mathbf{V}_{2}} u_{j}\right| \leq \varepsilon
$$

where $\varepsilon$ represents the permissible load-generation power unbalance within islands.

As it can be noticed in (5), the sectionalising strategy $S$ is then determined as the set of transmission lines to be left disconnected during the restoration process taking into consideration $\mathrm{BSC}$ and PBC within islands [7]. In (5), an AND logic gate is represented by the symbol $\otimes$.

$$
S=B S C \otimes P B C
$$

The sectionalising strategies obtained from (5) are then evaluated based on their voltage stability performance. Only one strategy which excludes critical transmission lines on the boundary of islands will be selected [7].

\section{B. Second step: reconstruction of the backbone}

The second step of the proposed method is separated into three sub-stages. This step is simultaneously carried out for each determined island i.e. islands are restored in parallel. The identification sub-stage determines multiple simple paths from the BS units to the NBS units and CLs in the same island. By using a $k$-shortest simple paths algorithm, these paths can be efficiently computed. Here, the number of connections required to energise the element at the destination node and the electrical distance from the source node to the destination node are computed for each path. In the simulation sub-stage, an Optimal Power Flow (OPF) algorithm, which optimises the active power losses and determines the optimal power system operating point for each possible path, is performed. Finally, the path with the minimum Total Weight Factor $(T W F)$ is executed in the implementation sub-stage. The availability of PMUs to monitor unpredictable events during PSR is assumed in the last sub-stage. This paper also assumes that the assessment of the power system status was previously carried out. In 


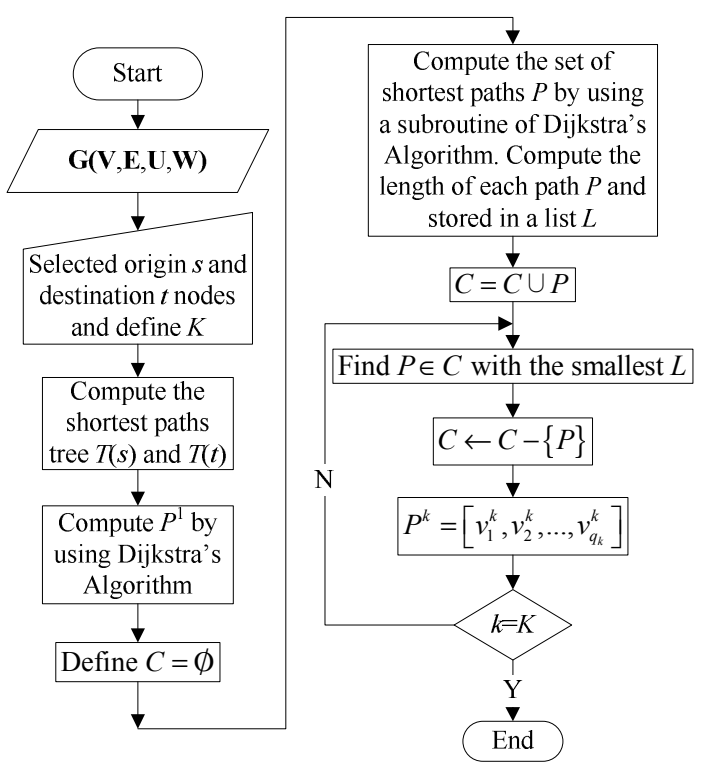

Fig. 4. Basic steps for $k$ shortest simple path algorithm

other words, the status of circuit breakers, power plant conditions, and local energy storage status are known [6].

1) Identification sub-stage: efficient algorithm for the $k$ shortest simple paths

The identification sub-stage in the reconstruction step applies an efficient algorithm to determine the $k$-shortest simple paths from the source node to the destination nodes.

Considering two designated nodes $s, t \in \mathbf{V}$, a path $P$ of $\mathbf{G}$ from a BS unit located at node $s$ to a NBS unit or CL located at node $t$ is an alternating sequence $v_{0}, e_{0}, v_{1}, e_{1}, \ldots, e_{n-1}, v_{n}$, of distinct nodes $v_{i} \in \mathbf{V}$ and edges $e_{i} \in \mathbf{E}$, where $v_{0}=s$ and $v_{n}=t$. The total length $w(P)$ from $s$ to $t$ in the path $P$ is defined as the sum of the symmetric lengths contained in $P$.

$$
w(P)=\sum_{i \in P} w_{i}
$$

Based on the given definition of $w_{i}$ in (3), when computing the shortest paths, the number of edges to be reconnected in the path $P$ from $s$ to $t$, is known. In parallel to the $k$-shortest simple paths computation, the total electrical distance between these two nodes across the paths can also be computed. Since the reactance $x_{i j}$ is commonly higher than the resistance $r_{i j}$, the latter is neglected when computing this electrical distance. Therefore, a new set $\mathbf{Z} \subset \mathbf{G}$, with elements $z_{i} \in \mathbf{Z}, i=1,2, \ldots, n_{l}$, representing the reactance $x_{i j}$ from the bus $i$ to the bus $j$, is defined. The total electrical distance from $s$ to $t$ in the paths $P$ is computed as follows.

$$
z(P)=\sum_{i \in P} z_{i}
$$

The problem of finding the shortest simple path from $s$ to $t$ is studied in [11]. However, considering all the problems that might arise during the restoration process, such as unavailability of branches, this paper implements an algorithm to determine the $k$-shortest simple paths [12]. Therefore, when the absolute shortest simple path cannot be implemented during the restoration process, the second or third shortest simple paths can be performed instead. Also, by determining various shortest simple paths, it is possible to monitor if $w(P)$ and $z(P)$ for each path change after the reconnection of a branch. Since connecting branches might change the number of connections to be required and the electrical distance to the elements which are not reconnected at this stage of the restoration process, it is possible that the second or third shortest simple paths firstly determined change when updating the shortest paths list from the BS units to the NBS units or CLs.

The proposed $k$-shortest simple paths algorithm uses a subroutine of the first simple path to determine the shortest simple path from $s$ to $t$. Then, a set of $k$-shortest simple paths $\left\{P^{1}, P^{2}, \ldots, P^{k-1}, P^{k}\right\}$ is determined. The algorithm to determine the $k$-shortest simple paths is presented in an ALGOL-like language in [12] and it is illustrated in a flow chart in Fig. 4.

2) Simulation sub-stage: Optimal power flow algorithm

When several shortest simple paths from the BS units to the NBS units or CLs are determined, and the number of branches $w(P)$ to be reconnected when energising the elements at all of the nodes $t$ and the correspondent electrical distance $z(P)$ are known, an OPF algorithm is run to optimise the active power losses and to determine the optimal operating point for each path.

The OPF algorithm is also used to calculate the voltage magnitude at bus $t$, denoted by $\left|V_{t}\right|$, and to monitor the static constraints, such as load-generation balance, transmission line loading, and voltage limits. These constraints are included in the OPF, which is formulated as follows.

$$
\begin{aligned}
& \min \quad f(\mathbf{x}) \\
& \text { subject to } \\
& \qquad \mathbf{g}(\mathbf{x})=0 \\
& \mathbf{h}_{\text {min }} \leq \mathbf{h}(\mathbf{x}) \leq \mathbf{h}_{\text {max }} \\
& \mathbf{x}_{\text {min }} \leq \hat{\mathbf{I}} \mathbf{x} \leq \mathbf{x}_{\text {max }}
\end{aligned}
$$

Here, $\mathbf{x}$ is the vector of optimisation variables, $f(\mathbf{x})$ is the objective function, $g(\mathbf{x})$ are the equality constraints, $h(\mathbf{x})$ are the inequality constraints bounded by $\mathbf{h}_{\min }$ and $\mathbf{h}_{\max }, \mathbf{x}_{\min }$ and $\mathbf{x}_{\max }$ represent respectively the minimum and maximum values of the parameters. The functions $f(\mathbf{x}), g(\mathbf{x})$ and $h(\mathbf{x})$ are assumed to be continuous and differentiable at least twice for $\mathbf{x} \in \mathbf{R}^{n_{\mathbf{x}}}$. A primal-dual interior point approach [13] is used to solve (8). The vector $\mathbf{x}$ is constituted by two $n_{b} \times 1$ vectors of voltage magnitudes and phases and two $n_{g} \times 1$ vectors of active and reactive power generation.

$$
\mathbf{x}=\left[\begin{array}{llll}
\mathbf{v} & \boldsymbol{\theta} & \mathbf{P}_{\mathrm{g}} & \mathbf{Q}_{\mathrm{g}}
\end{array}\right]^{T}
$$

As the capacity of the branches is considerably important, the objective function in (8) is represented by the total active power losses. This optimises the power flow in branches, and therefore, sufficient capacity is ensured during the restoration process. The objective function is modelled as follows.

$$
\min f=\min \sum_{i=1}^{n_{b}} \sum_{j=1}^{n_{b}} g_{i j}\left(V_{i}^{2}+V_{j}^{2}-2 V_{i} V_{j} \cos \left(\theta_{i}-\theta_{j}\right)\right)
$$

where $g_{i j}$ is the series susceptance between any two connected buses $i$ and $j$. The bus voltage magnitudes and phases at these buses are represented by $V_{i}, V_{j}, \theta_{i}$ and $\theta_{j}$, respectively.

The power balance equations, for the active and reactive power, can be formulated in terms of voltage magnitudes and phases, power load and power generation. Thus, the equality constraints in (8) are computed as follows. 


$$
\begin{gathered}
\mathbf{g}_{\mathbf{P}}\left(\mathbf{v}, \boldsymbol{\theta}, \mathbf{P}_{\mathrm{g}}\right)=\mathbf{P}_{\mathrm{i}}(\mathbf{v}, \boldsymbol{\theta})+\mathbf{P}_{\mathrm{d}}-\mathbf{P}_{\mathrm{g}} \\
\mathbf{g}_{\mathbf{Q}}\left(\mathbf{v}, \boldsymbol{\theta}, \mathbf{Q}_{\mathrm{g}}\right)=\mathbf{Q}_{\mathrm{i}}(\mathbf{v}, \boldsymbol{\theta})+\mathbf{Q}_{\mathrm{d}}-\mathbf{Q}_{\mathrm{g}}
\end{gathered}
$$

where, $\mathbf{P}_{\mathbf{i}}$ and $\mathbf{Q}_{\mathrm{i}}$ are the vectors of active and reactive power injections at the bus, $\mathbf{P}_{\mathbf{d}}$ and $\mathbf{Q}_{\mathbf{d}}$ are the vectors of active and reactive power load at the bus, and $\mathbf{P}_{\mathrm{g}}$ and $\mathbf{Q}_{\mathrm{g}}$ are the vectors of active and reactive power generation at the bus.

Overloads in branches are an important concern during PSR. Thus, a double set of $n_{l}$ branch flow limits is considered in this paper as an inequality constraint. These two sets are functions of the bus voltage magnitudes and phases, one for the sending end $i\left(\mathbf{P}_{\mathbf{i j}}\right.$ and $\left.\mathbf{Q}_{\mathrm{ij}}\right)$ and one for the receiving end $j\left(\mathbf{P}_{\mathbf{j i}}\right.$ and $\left.\mathbf{Q}_{\mathbf{j i}}\right)$ of each branch.

$$
\begin{aligned}
& \mathbf{h}_{\mathrm{ij}}(\mathbf{v}, \boldsymbol{\theta})=\mathbf{F}_{\max }-\left|\mathbf{P}_{\mathrm{ij}}(\mathbf{v}, \boldsymbol{\theta})+j \mathbf{Q}_{\mathrm{ij}}(\mathbf{v}, \boldsymbol{\theta})\right| \geq \mathbf{0} \\
& \mathbf{h}_{\mathrm{ji}}(\mathbf{v}, \boldsymbol{\theta})=\mathbf{F}_{\max }-\left|\mathbf{P}_{\mathrm{ji}}(\mathbf{v}, \boldsymbol{\theta})+j \mathbf{Q}_{\mathrm{ji}}(\mathbf{v}, \boldsymbol{\theta})\right| \geq \mathbf{0}
\end{aligned}
$$

The optimisation variables are bounded by upper and lower thresholds which impose the limits for these variables. Thus, the vectors $\mathbf{x}_{\min }$ and $\mathbf{x}_{\max }$ are represented as follows.

$$
\begin{array}{ll}
\mathbf{v}_{\mathbf{i}, \text { min }} \leq \mathbf{v}_{\mathbf{i}} \leq \mathbf{v}_{\mathbf{i}, \text { max }} & \text { for all } i \in \mathbf{V} \\
\boldsymbol{\theta}_{\mathbf{i}, \text { min }} \leq \boldsymbol{\theta}_{\mathbf{i}} \leq \boldsymbol{\theta}_{\mathbf{i}, \text { max }} & \text { for all } i \in \mathbf{V} \\
\mathbf{P}_{\mathbf{g}, \mathbf{i} \text { min }} \leq \mathbf{P}_{\mathbf{g}, \mathbf{i}} \leq \mathbf{P}_{\mathbf{g}, \mathbf{i} \text { max }} & \text { for all } i \in \mathbf{V}_{\mathbf{G}} \\
\mathbf{Q}_{\mathbf{g}, \mathbf{i} \text { min }} \leq \mathbf{Q}_{\mathbf{g}, \mathbf{i}} \leq \mathbf{Q}_{\mathbf{g}, \mathbf{i} \text { max }} & \text { for all } i \in \mathbf{V}_{\mathbf{G}}
\end{array}
$$

After running the implemented OPF, the steady-state voltage magnitude at bus $t,\left|V_{t}\right|$, is computed and used as a weight factor when selecting the first element to restore. Since the value of $\left|V_{t}\right|$ is bounded (15) and is normally close to the nominal value, the $T W F$ is calculated then as follows.

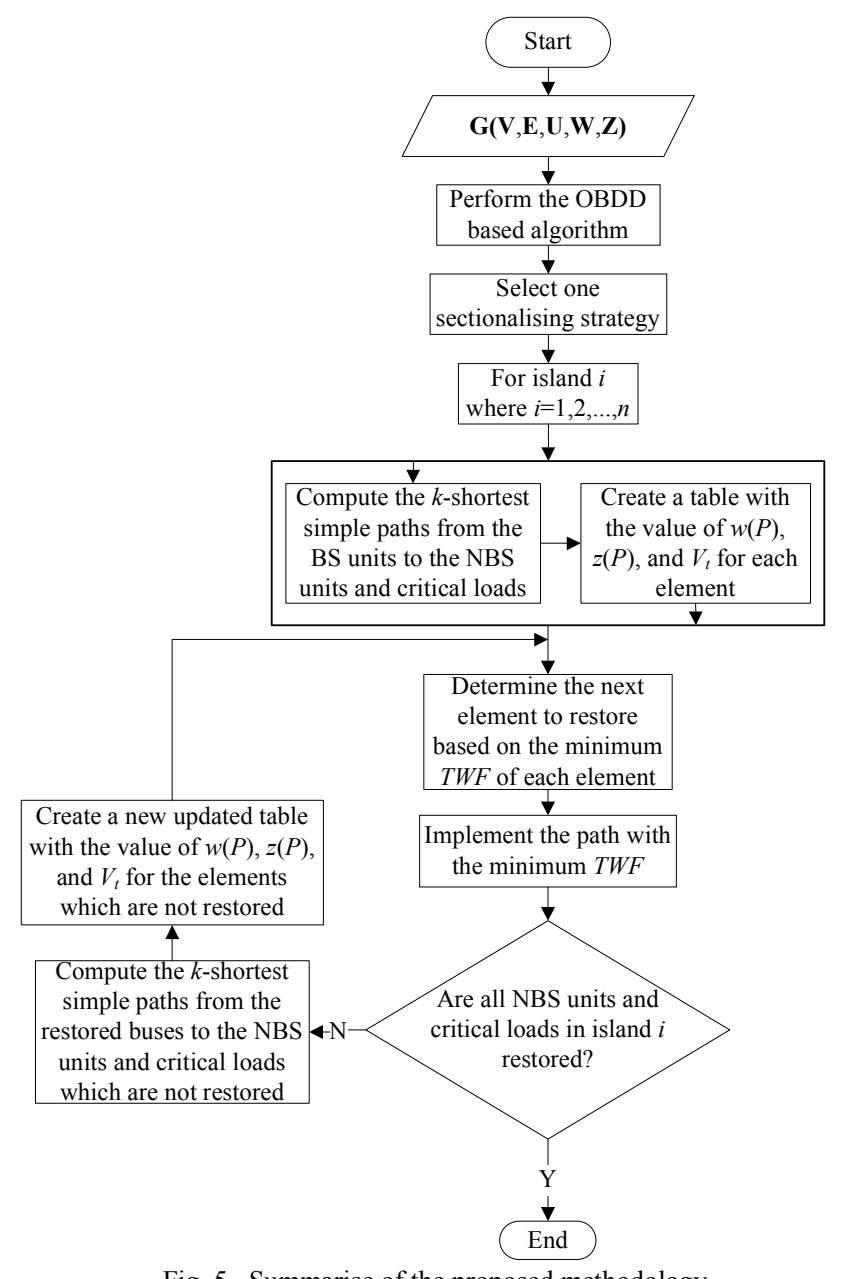

Fig. 5. Summarise of the proposed methodology

$$
T W F= \begin{cases}\frac{w(P) \cdot z(P)}{\left|V_{t}\right|} & \text { if }\left|V_{t}\right|<1 \\ w(P) \cdot z(P) \cdot\left|V_{t}\right| & \text { if }\left|V_{t}\right| \geq 1\end{cases}
$$

As the restoration time duration is highly important and must be minimised, the path with the minimum $T W F$ is implemented in the next sub-stage.

3) Implementation sub-stage: final stage to restore the power system

When the $T W F$ for the $k$-shortest simple paths from the BS units to all NBS units and CLs are computed, the case with minimum $T W F$ is selected. At this final sub-stage of the second step, the instructions to implement the optimal path, based on operators experience and solution obtained, are performed. Since the system topology and operating point change when implementing a selected path, it is important to update the solutions previously obtained after each path implementation i.e. the solution proposed is now converted into an iterative process which updates previous solutions and stops when all elements are restored.

The use of PMUs is essential during the implementation stage to monitor and to help on controlling high voltages, unexpected large charging currents and other factors that might be encountered during PSR.

\section{Summarise of the proposed method}

The proposed method can be summarised as shown in Fig. 5. As noticed, the only input to the method is the graph information presented in Section III. Then, the OBDD based algorithm determines one sectionalising strategy to separate the entire network into two islands. When these islands are known, the second step of the proposed method is carried out in parallel for all the created islands. For each island, the three sub-stages are then performed. The $k$-shortest simple paths from the BS units to all the NBS units and CLs are computed first. These paths are stored in a table with the corresponding values of $w(P), z(P)$ and $\left|V_{t}\right|$. The element to restore is based on the smallest $T W F$. When this element is restored, a new updated table is computed with the new values of $w(P), z(P)$ and $\left|V_{t}\right|$ from the restored buses to the remaining elements which are not restored at this stage of the restoration process. This last step is repeated until all NBS units and CLs in island $i=1,2, \ldots n$ are in service.

\section{SimULATION RESUltS}

The proposed methodology is tested and implemented on the IEEE 118-bus test system. This test system contains 54 synchronous machines (19 generators and 35 condensers), 177 transmission lines, 9 transformers and 91 constant power loads [14]. The topology of the system can be seen in Fig. 6. This paper considers only two BS units. These machines, located at buses 25 and 69 , are the two source nodes in the power system. Given the location of the sources, and based on the BSC, only two islands can be created. The remaining generators are considered as NBS units. CLs are assumed to be located at buses 15, 18, 23, 27, $49,54,59,80,90$, and 92 . Table I presents the time required to complete an action during PSR. As it can be noticed, these times are expressed as a variable which can be later changed according to the restoration plan for each network.

In the first step of the proposed method, the OBDD based 


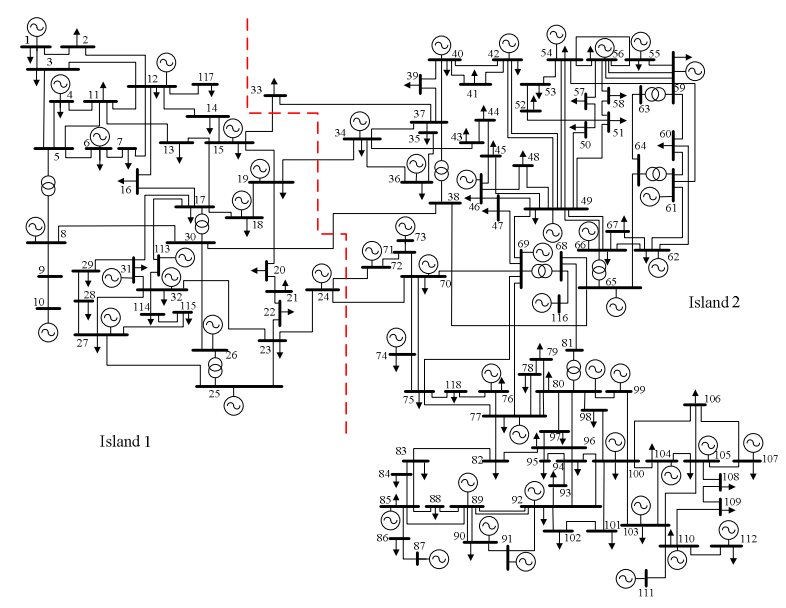

Fig. 6. Sectionalising strategy obtained in the first step

method explained in Section III-A is used to determine the sectionalising strategy i.e. the set of transmission lines to be left disconnected during the restoration process in order to sectionalise the network into different islands.

To reduce the number of possible sectionalising strategies and to obtain a smaller active power unbalance, this paper considers a maximum load-generation unbalance $\varepsilon$ of $100 \mathrm{MW}$. This paper assumes the active power generation as shown in the steady-state operation [14]. Given this information, the OBDD based method is carried out and multiple sectionalising strategies are obtained. Fig. 6 presents one of these sectionalising strategies obtained when separating the IEEE 118-bus into two islands after the blackout. As noticed, $S=\{(15,33),(19,34),(30,38),(24,70)$, $24,72)\}$. Since the BSC and PBC are included in the algorithm, each island contains one BS unit and the loadgeneration unbalance is equal or smaller than $\varepsilon$. The actual unbalance and the CLs within islands are shown in Table II.

TABLE I

TIME TO COMPLETE AN ACTION DURING PSR

\begin{tabular}{lc}
\hline \hline \multicolumn{1}{c}{ Actions } & Time $(\mathrm{min})$ \\
\hline \hline Restart BS unit & $t_{B S}$ \\
\hline Energise a bus from BS unit & $t_{b}$ \\
\hline Connect tie line & $t_{t l}$ \\
\hline Crank power to a NBS unit from a bus & $t_{N B S}$ \\
\hline Synchronised subsystems & $t_{s y n}$ \\
\hline Pick up load & $t_{p u}$ \\
\hline \hline
\end{tabular}

TABLE II

TOTAL LOAD AND GENERATION WITHIN ISLAND FOR THE TEST SYSTEM

\begin{tabular}{ccccc}
\hline \hline $\begin{array}{c}\text { Island } \\
\text { No. }\end{array}$ & $\begin{array}{c}\Sigma P_{G} \\
(M W)\end{array}$ & $\begin{array}{c}\Sigma P_{L} \\
(M W)\end{array}$ & $\begin{array}{c}\Sigma\left(P_{G}-P_{L}\right) \\
(M W)\end{array}$ & CLs \\
\hline \hline 1 & 1076.0 & 976.0 & 100.0 & $15,18,23,27$ \\
\hline 2 & 3301.4 & 3266.0 & 35.4 & $49,54,59,80,90,92$ \\
\hline \hline
\end{tabular}

By separating the system into only two islands, the stability margins would be sufficiently high during PSR. Also, as previously mentioned, one BS unit is included in each island i.e. generators at bus 25 and 69 for the Island 1 and Island 2, respectively. The NBS units in the Island 1 are located at buses $10,12,26$, and 31 . Whilst the NBS units in the Island 2 are located at buses 46, 49, 54, 59, 61, 65, 66, $80,87,89,100,103$, and 111 . For the next step, the thermal ramping is not considered. However, by using the times shown in Table I, this value can be taken into account.

\section{A. Results for the second step}

When one sectionalising strategy to separate the network into two subsystems is known, the reconstruction of these subsystems starts. Due to space limitation, the second step of the method is explained in detail using the Island 1.

As it was previously explained, the identification substage is first carried out. The $k$ shortest simple paths from the source node, the BS unit located at bus 25, to the destination nodes, the NBS units and CLs, are then determined. In this paper, $k$ is set to be three. With this value of $k$, path redundancy, defined as multiple paths from $s$ to $t$, is ensured in case of unexpected unavailability of branches.

The 3-shortest simple paths to the NBS units and CLs in the Island 1 and the Island 2 are shown in Table III and Table IV, respectively. When similar number of branch connections is obtained in different paths, the electrical distance is used as the second criterion to select only the 3shortest simple paths.

TABLE III

ThreE SHORTEST PATHS TO OTHER ELEMENTS IN THE ISLAND 1

\begin{tabular}{|c|c|c|c|c|}
\hline$s$ & $t$ & Path & $\begin{array}{l}w(P) \\
(p . u .)\end{array}$ & $\begin{array}{c}z(P) \\
(\text { p.u. })\end{array}$ \\
\hline \multirow{3}{*}{25} & \multirow{3}{*}{10} & {$\left[\begin{array}{l}{[25,26,30,8,9,10]} \\
\end{array}\right.$} & 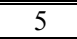 & 0.2373 \\
\hline & & {$[25,23,32,113,17,30,8,9,10]$} & 8 & 0.5803 \\
\hline & & {$[25,23,32,31,17,30,8,9,10]$} & 8 & 0.6020 \\
\hline \multirow{3}{*}{25} & \multirow{3}{*}{12} & {$[25,26,30,17,16,12]$} & 5 & 0.4265 \\
\hline & & {$[25,26,30,8,5,11,12]$} & 6 & 0.2891 \\
\hline & & {$[25,26,30,8,5,3,12]$} & 6 & 0.4693 \\
\hline \multirow{3}{*}{25} & \multirow{3}{*}{15} & {$[25,26,30,17,15]$} & 4 & 0.2067 \\
\hline & & {$[25,23,32,113,17,15]$} & 5 & 0.4721 \\
\hline & & {$[25,23,32,31,17,15]$} & 5 & 0.4938 \\
\hline \multirow{3}{*}{25} & \multirow{3}{*}{18} & {$[25,26,30,17,18]$} & 4 & 0.2135 \\
\hline & & {$[25,23,32,113,17,18]$} & 5 & 0.4789 \\
\hline & & {$[25,23,32,31,17,18]$} & 5 & 0.5006 \\
\hline \multirow{3}{*}{25} & \multirow{3}{*}{23} & {$[25,23]$} & 1 & 0.0800 \\
\hline & & {$[25,27,32,23]$} & 3 & 0.3538 \\
\hline & & {$[25,27,115,114,32,23]$} & 5 & 0.4240 \\
\hline \multirow{3}{*}{25} & \multirow{3}{*}{26} & {$[25,26]$} & 1 & 0.0382 \\
\hline & & {$[25,23,32,113,17,30,26]$} & 6 & 0.5532 \\
\hline & & {$[25,23,32,31,17,30,26]$} & 6 & 0.5749 \\
\hline \multirow{3}{*}{25} & \multirow{3}{*}{27} & {$[25,27]$} & 1 & 0.1630 \\
\hline & & {$[25,23,32,27]$} & 3 & 0.2708 \\
\hline & & {$[25,23,32,114,115,27]$} & 5 & 0.3410 \\
\hline \multirow{3}{*}{25} & \multirow{3}{*}{31} & {$[25,23,32,31]$} & 3 & 0.2938 \\
\hline & & {$[25,27,32,31]$} & 3 & 0.3370 \\
\hline & & {$[25,26,30,17,31]$} & 4 & 0.3193 \\
\hline
\end{tabular}

Since $\left|V_{t}\right|$ is normally close to the nominal value, it can be noticed from (16) that the most favourable path depends directly on the number of connections required to connect the element and the electrical distance to this element. For the Island 1, it can be noticed in Table III that three possible elements can be energised first. These elements, located at buses 23,26 or 27 , only require one branch connection to be energised. However, as the electrical distance from bus 25 to bus 26 is smaller than the others, cranking power from the BS unit to this NBS unit is sent through edge $(25,26)$ first. By running the OPF algorithm, it can be determined that the steady-state voltage $\left|V_{26}\right|=0.975$ p.u. By analysing the steps to follow during the restoration process and using Table I, it can be concluded that this action is accomplished after $t_{B S}+t_{b}+t_{N B S}$.

Since the second shortest element is located at bus 23 , the load at this bus should be picked up second. When picking up this load and by using the OPF algorithm previously explained, $\left|V_{23}\right|=1.060$ p.u. is obtained. Since the previous two elements are restored, the third element to be restored is consequently the load at bus 27 . When picking up this load, it is obtained that $\left|V_{27}\right|=1.044$ p.u. These last two actions are accomplished after $2 t_{b}+2 t_{p u}$. As seen in Table III, the shortest simple paths previously determined are highly 
dependent on the previous restore paths ([25,23], [25,26] and $[25,27])$. Therefore, the number of connections to restore the remaining elements in the Island 1 is reduced when these three elements are restored.

TABLE IV

Three SHORTESt PATHS to OTHER ELEMENTS IN THE ISLAND 2

\begin{tabular}{|c|c|c|c|c|}
\hline$s$ & $t$ & Path & $\begin{array}{l}w(P) \\
(p . u .) \\
\end{array}$ & $\begin{array}{c}z(P) \\
(p . u .) \\
\end{array}$ \\
\hline \multirow{3}{*}{69} & \multirow{3}{*}{46} & {$[69,47,46]$} & 2 & 0.4048 \\
\hline & & {$[69,49,45,46]$} & 3 & 0.6456 \\
\hline & & {$[69,49,47,46]$} & 3 & 0.5135 \\
\hline \multirow{3}{*}{69} & \multirow{3}{*}{49} & {$[69,49]$} & 1 & 0.3240 \\
\hline & & {$[69,47,49]$} & 2 & 0.3403 \\
\hline & & {$[69,68,65,66,49]$} & 4 & 0.1819 \\
\hline \multirow{3}{*}{69} & \multirow{3}{*}{54} & {$[69,49,54]$} & 2 & 0.6150 \\
\hline & & {$[69,47,49,54]$} & 3 & 0.6313 \\
\hline & & {$[69,68,65,66,49,54]$} & 5 & 0.4729 \\
\hline \multirow{3}{*}{69} & \multirow{3}{*}{59} & {$[69,49,54,59]$} & 3 & 0.8443 \\
\hline & & {$[69,47,49,54,59]$} & 4 & 0.8606 \\
\hline & & {$[69,49,54,56,59]$} & 4 & 0.8635 \\
\hline \multirow{3}{*}{69} & \multirow{3}{*}{61} & {$[69,49,54,59,61]$} & 4 & 0.9943 \\
\hline & & {$[69,68,65,64,61]$} & 4 & 0.1100 \\
\hline & & {$[69,49,66,62,61]$} & 4 & 0.6715 \\
\hline \multirow{3}{*}{69} & \multirow{3}{*}{65} & {$[69,68,65]$} & 2 & 0.0530 \\
\hline & & {$[69,49,66,65]$} & 3 & 0.4529 \\
\hline & & {$[69,47,49,66,65]$} & 4 & 0.4692 \\
\hline \multirow{3}{*}{69} & \multirow{3}{*}{66} & {$[69,49,66]$} & 2 & 0.4159 \\
\hline & & {$[69,47,49,66]$} & 3 & 0.4322 \\
\hline & & {$[69,68,65,66]$} & 3 & 0.0900 \\
\hline \multirow{3}{*}{69} & \multirow{3}{*}{80} & {$[69,77,80]$} & 2 & 0.2060 \\
\hline & & {$[69,75,77,80]$} & 3 & 0.4269 \\
\hline & & {$[69,68,81,80]$} & 3 & 0.0942 \\
\hline \multirow{3}{*}{69} & \multirow{3}{*}{87} & {$[69,77,82,83,85,86,87]$} & 6 & 0.7014 \\
\hline & & {$[65,75,77,82,83,85,86,87]$} & 7 & 0.9223 \\
\hline & & {$[69,77,82,83,84,85,86,87]$} & 7 & 0.7494 \\
\hline \multirow{3}{*}{69} & \multirow{3}{*}{89} & {$[69,77,82,83,85,89]$} & 5 & 0.5439 \\
\hline & & {$[69,77,82,83,85,88,89]$} & 6 & 0.5442 \\
\hline & & {$[69,77,82,83,84,85,89]$} & 6 & 0.5920 \\
\hline \multirow{3}{*}{69} & \multirow{3}{*}{90} & {$[69,77,82,83,85,89,90]$} & 6 & 0.6436 \\
\hline & & {$[69,77,82,83,85,88,89,90]$} & 7 & 0.6439 \\
\hline & & {$[69,77,82,83,84,85,89,90]$} & 7 & 0.6918 \\
\hline \multirow{3}{*}{69} & \multirow{3}{*}{92} & {$[69,77,82,96,94,92]$} & 5 & 0.4842 \\
\hline & & {$[69,77,80,96,94,92]$} & 5 & 0.6329 \\
\hline & & {$[69,77,80,98,100,92]$} & 5 & 0.7880 \\
\hline & & {$[69,77,80,98,100]$} & 4 & 0.4930 \\
\hline 69 & 100 & {$[69,77,80,99,100]$} & 4 & 0.4933 \\
\hline & & {$[69,68,81,80,98,100]$} & 5 & 0.3812 \\
\hline & & {$[69,77,80,98,100,103]$} & 5 & 0.5455 \\
\hline 69 & 103 & {$[69,77,80,99,100,103]$} & 5 & 0.5458 \\
\hline & & {$[69,68,81,80,98,100,103]$} & 6 & 0.4337 \\
\hline & & {$[69,77,80,98,100,103,110,111]$} & 7 & 0.8023 \\
\hline 69 & 111 & {$[69,77,80,99,100,103,110,111]$} & 7 & 0.8026 \\
\hline & & {$[69,68,81,80,98,100,103,110,111]$} & 8 & 0.6905 \\
\hline
\end{tabular}

After updating Table III, it can be noticed that the next element to energise is the NBS unit located at bus 31 . To crank power to this NBS unit, only two transmission lines connections are required. This can be noticed from the first two paths shown in Table III i.e. [25,23,32,31] and $[25,27,32,31]$. Since the branches $(25,23)$ and $(25,27)$ were previously restored, it is important to compute the new electrical distance. It can be noticed that the source node is still the BS unit, but the branches to be restored are only $(23,32)$ and $(32,31)$ or $(27,32)$ and $(32,31)$, respectively for each path. Since the electrical distance is similar, the steadystate voltage for each path is calculated to rank these possible paths. When implementing the path [23,32,31], the steady- state voltage is $\left|V_{31}\right|=1.056$ p.u. On the other hand, when implementing the path [27,32,31], this value is $\left|V_{31}\right|=0.967$ p.u. By comparison of these two possible paths, it can be noticed that the first mentioned path posses a higher $T W F$ in comparison to the second path. In the implementation sub-stage, therefore, the latter path is carried out. Here, the availability of all involved branches $(27,32)$ and $(32,31)$ is assumed. The NBS unit located at bus 31 is then in operation after $2 t_{b}+t_{N B S}$.

When completing the previous action, Table III must be updated again to determine the next optimal action. Following this, it can be noted that paths $[31,17,15]$ and $[31,17,18]$ allow the reconnection of CLs located at bus 15 and 18 , in only two actions, respectively. In addition to the number of connections done to pick up these loads, the electrical distance from the source node (in this case generator at bus 31) to these elements is similar and the next parameter to take into account is the steady-state voltage obtained at bus 15 and 18. By running the OPF previously explained, it can be obtained that $\left|V_{15}\right|=0.999$ p.u. and $\left|V_{18}\right|=1.022$ p.u. By applying (16), it is noted that the $T W F$ is lower when picking up the load at bus 15 . Thus, this load is picked up first and the load at 18 should be picked up second. These elements are connected after $3 t_{b}+2 t_{p u}$.

By picking up these two loads in the mentioned sequence, all CLs are now synchronised and only two NBS units are needed to be cranked in the Island 1. Similar to previous steps, it is now necessary to update Table III. By updating this table, it can be noticed that the NBS unit at bus 12 can be cranked by connecting edges $(17,16)$ and $(16,12)$. This NBS unit is connected after $2 t_{b}+t_{N B S}$ and the optimal voltage is $\left|V_{12}\right|=1.060$ p.u.

Finally for the Island 1, the shortest paths to crank power to the NBS unit located at bus 10 are updated. It can be noted, by using the path $[17,30,8,9,10]$ or $[26,30,8,9,10]$, the same number of connections is obtained, and therefore, the electrical distance and the optimal voltage when using each path is computed. By running the presented OPF, the voltage at bus 10 is 1.030 p.u. and 1.041 p.u. respectively for the paths previously mentioned. In addition, it can be calculated that the electrical distance is shorter by using the first path. Therefore, after $4 t_{b}+t_{N B S}$ more, it can be noted that the NBS unit at bus 10 is connected by using the path $[17,30,8,9,10]$.

By adding up all the times required to restore the mentioned elements in the Island 1, it can be noticed that the CLs and NBS units are all synchronised after a total time of $t_{B S}+4 t_{N B S}+14 t_{b}+4 t_{p u}$. By using the times presented in [15], this total time represents $185 \mathrm{~min}$.

Although the restoration actions for the Island 2 are analysed in a second instance, these actions are performed in parallel to the restoration actions previously presented for the Island 1. NBS units and CLs in the Island 2 were previously mentioned and the 3 -shortest simple paths to these elements are presented in Table IV.

By performing a similar analysis as presented for the Island 1, but now in the Island 2, the optimal paths from the source nodes, the BS unit at bus 69, to the NBS units and CLs can be obtained. Table $\mathrm{V}$ presents, decreasingly, the optimal restoration sequence and the most favourable path.

As it can be noticed in Table $\mathrm{V}$, the elements to be restored first are located at bus 49. A CL and a NBS unit are located at this bus. The optimal path and the steady-state voltage to energise these elements are shown in Table V. By using the explained OPF algorithm, it can be noticed that the 
steady-state voltage at bus 49 is $1.026 \mathrm{p.u}$. These elements are restored after $t_{B S}+t_{b}+t_{p u}+t_{N B S}$. After performing the above action, the number of connections, to restore the remaining elements in the Island 2, changes.

When bus 49 is energised, it can be noticed that the next element to be restored is the NBS unit located at bus 66 (see Table IV and V for reference). In order to crank power to this unit, only one branch connection, from generator 49 , is now required. This unit is in operation after $t_{b}+t_{N B S}$.

TABLE V

RESTORATION ACTIONS TO RESTORE THE ISLAND 2

\begin{tabular}{cccccc}
\hline \hline \multirow{2}{*}{$s$} & $t$ & Path & $\begin{array}{c}w(P) \\
(p . u .)\end{array}$ & $\begin{array}{c}z(P) \\
(p . u .)\end{array}$ & $\begin{array}{c}\left|V_{t}\right| \\
(p . u .)\end{array}$ \\
\hline \hline 69 & 49 & {$[69,49]$} & 1 & 0.3240 & 1.026 \\
\hline 49 & 66 & {$[49,66]$} & 1 & 0.0919 & 1.046 \\
\hline 66 & 65 & {$[66,65]$} & 1 & 0.0370 & 0.982 \\
\hline 49 & 54 & {$[49,54]$} & 1 & 0.2910 & 1.026 \\
\hline 54 & 59 & {$[54,59]$} & 1 & 0.2293 & 1.041 \\
\hline 49 & 46 & {$[49,47,46]$} & 2 & 0.1895 & 1.057 \\
\hline 69 & 80 & {$[69,77,80]$} & 2 & 0.2060 & 1.048 \\
\hline 66 & 61 & {$[66,62,61]$} & 2 & 0.2556 & 1.060 \\
\hline 80 & 100 & {$[80,98,100]$} & 2 & 0.2870 & 1.060 \\
\hline 100 & 103 & {$[100,103]$} & 1 & 0.0525 & 1.060 \\
\hline 100 & 92 & {$[100,92]$} & 1 & 0.2950 & 0.959 \\
\hline 103 & 111 & {$[103,110,111]$} & 2 & 0.2568 & 1.059 \\
\hline 80 & 89 & {$[77,82,83,85,89]$} & 4 & 0.4430 & 1.005 \\
\hline 89 & 90 & {$[89,90]$} & 1 & 0.0997 & 0.985 \\
\hline 89 & 87 & {$[85,86,87]$} & 2 & 0.3304 & 1.015 \\
\hline \hline
\end{tabular}

Following this, and by using the second shortest path shown in Table IV to the NBS unit at bus 65 , it is noted that this NBS unit is restored by reconnecting only the branch $(66,65)$. Here, it is important to note the importance of determining multiple paths. For this particular case, the absolute shortest path from 69 to $65([69,68,65])$ is actually now relegated by the path $[66,65]$. This NBS unit is in operation after $t_{b}+t_{N B S}$.

By following the same analysis, it can be noticed that all the elements in the Island 2 are restored after a total time of $t_{B S}+24 t_{b}+6 t_{p u}+13 t_{N B S}$. By using the times presented in [15], this total time represents $390 \mathrm{~min}$.

It is important to note that when all units are synchronised within the islands, the remaining elements can be then restored by using the same method presented above i.e. by applying the proposed method summarised in Fig. 5. Here, it is important to point out that load pick up must be carried out in small increments to avoid excessive frequency deviations.

\section{CONCLUSION}

Merging the two main strategies to restore the power system after a blackout is proposed in this paper. The proposed method is based on two steps: sectionalisation and reconstruction of the network. In the first step, the method separates the network into the fewest areas possible to maintain the stability margins as high as possible. The second step is divided into three stages: identification, simulation and implementation. An efficient algorithm to determine the $k$ shortest simple paths from the blackstart unit to the remaining elements in the network is used in the first stage. Then, these options are ranked based on the number of connections required to energise the element, the electrical distance and the optimal steady-state voltage magnitude obtained from the developed OPF. In the final stage, implementation, the best option is selected and implemented in the power system. Simulations results demonstrate the effectiveness of the method to restore the network quickly.

\section{REFERENCES}

[1] IEEE PES Power System Engineering Committee, "Special considerations in power system restoration," IEEE Transactions on Power Systems, vol. 7, no. 4, pp. 1419-1427, Nov. 1992

[2] M.M. Adibi, et al., "Power system restoration - A task force report," IEEE Transactions on Power Systems, vol. 2, no. 2, pp. 271-277, May 1987.

[3] M.M. Adibi and N. Martins, "Power system restoration dynamic issues," in Proc. IEEE PES General Meeting, Pittsburgh, PA, Jul. 2008.

[4] N. Voropai and T. Hammons, "Blackouts: Remedial measures and restoration practices - Asian and Australian experience," in Proc. IEEE PES General Meeting, Pittsburgh, PA, Jul. 2008.

[5] R. Anjan, P. Pentayya and S.A. Khaparde, "Experience of blackouts and restoration practices in western region of India," in Proc. IEEE PES General Meeting, Denver, CO, Jun. 2004.

[6] M.M. Adibi and L.H. Fink, "Power system restoration planning," IEEE Transactions on Power Systems, vol. 9, no. 1, pp. 22-28, Feb. 1994.

[7] C. Wang, V. Vittal and K. Sun, "OBDD-based sectionalizing strategies for parallel power system restoration," IEEE Transactions on Power Systems, vol. 26, no. 3, pp. 1426-1433, Aug. 2011.

[8] Y. Liu and X. Gu, "Skeleton-network reconfiguration based on topological characteristics of scale-free networks and discrete particle swarm optimization," IEEE Transactions on Power Systems, vol. 22, no. 3, pp. 1267-1274, Aug. 2007.

[9] C. Wang, V. Vittal, V.S Kolluri and S. Mandal, "PTDF-based automatic restoration path selection," IEEE Transactions on Power Systems, vol. 25, no. 3, pp. 1686-1695 Aug. 2010.

[10] L.H. Fink, K.L. Liou and C.C. Liu, "From generic restoration actions to specific restoration strategies," IEEE Transactions on Power Systems, vol. 10, no. 2, pp. 745-751, May 1995.

[11] E.W. Dijkstra, "A note on two problems in connexion with graphs," Numerische mathematik, vol. 1, pp. 269-271, 1959.

[12] N. Katoh, T. Ibaraki and H. Mine, "An efficient algorithm for $k$ shortest simple paths," Networks, vol. 12, pp. 411-427, 1982.

[13] S.J. Wright, "Primal-Dual Interior-Point Methods," SIAM, 1997.

[14] University of Washington, "Power system test case archive," available at: http://www.ee.washington.edu/research/pstca/, consulted in Oct. 2010.

[15] J.S. Wu, C.C. Liu, K.L. Liou and R.F. Chu, "A petri net algorithm for scheduling of generic restoration actions," IEEE Transactions on Power Systems, vol. 12, no. 1, pp. 69-76, Feb. 1997.

\section{BIOGRAPHIES}

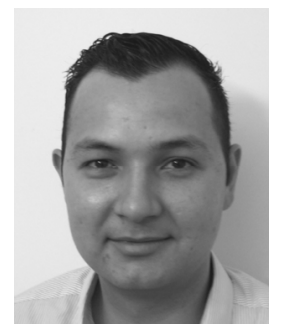

Jairo H. Quirós Tortós (S'08) was born in Limón, Costa Rica. He obtained the B.Sc. and Licentiate degree with honours in Electrical Engineering from the University of Costa Rica, San Pedro, Costa Rica in 2008 and 2009 respectively. Currently he is a Ph.D. student at The University of Manchester, working on power system restoration and intentional controlled islanding for power system. His main research interests are application of intelligent methods to power system restoration, and intentional controlled islanding, power system monitoring, protection and control, voltage stability assessment, and power system dynamics. At the end of his Ph.D. studies, he hopes to return to the University of Costa Rica as a Professor and Researcher.

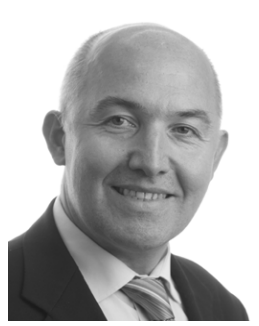

Vladimir Terzija (M'95, SM'2000) is the EPSRC Chair Professor in Power System Engineering in the School of Electrical and Electronic Engineering, The University of Manchester, where he has been since 2006. From 1997 to 1999, he was an Assistant Professor at the University of Belgrade. In 1999, he was awarded a prestigious Humboldt Research Fellowship. From 2000 to 2006, he was with ABB AG, Germany, working as an expert for switchgear and distribution automation. His main research interests are application of intelligent methods to power system monitoring, control, and protection, switchgear and fast transient processes, as well as DSP applications in power systems. 